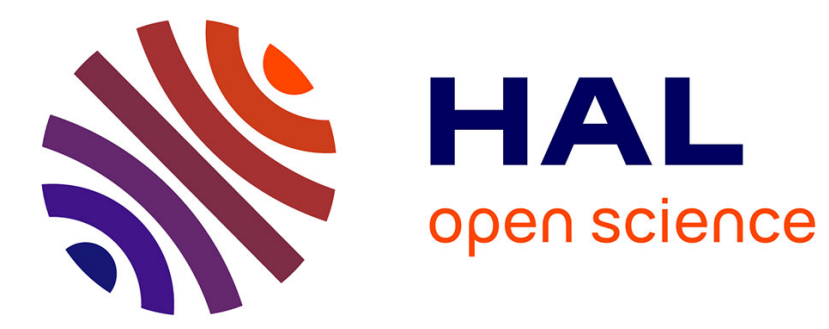

\title{
Radiative models for architectural modeling
}

Michaël Chelle, Bruno Andrieu

\section{To cite this version:}

Michaël Chelle, Bruno Andrieu. Radiative models for architectural modeling. Agronomie, 1999, 19 (3-4), pp.225-240. hal-00885926

\section{HAL Id: hal-00885926 https://hal.science/hal-00885926}

Submitted on 1 Jan 1999

HAL is a multi-disciplinary open access archive for the deposit and dissemination of scientific research documents, whether they are published or not. The documents may come from teaching and research institutions in France or abroad, or from public or private research centers.
L'archive ouverte pluridisciplinaire $\mathbf{H A L}$, est destinée au dépôt et à la diffusion de documents scientifiques de niveau recherche, publiés ou non, émanant des établissements d'enseignement et de recherche français ou étrangers, des laboratoires publics ou privés. 


\title{
Review article
}

\section{Radiative models for architectural modeling}

\author{
Michaël Chelle*, Bruno Andrieu \\ U. R. de Bioclimatologie, Inra, 78850 Thiverval-Grignon, France
}

(Received 10 November 1998; accepted 2 March 1999)

\begin{abstract}
Light microclimate determines many aspects of plant growth. Introducing the light-vegetation interactions into a plant model requires a radiative model computing fluxes at the spatial scale described in the plant model. The threedimensional (3D) architectural models describe plants functionally and geometrically as sets of interconnected organs. As the geometry of each organ is defined, the 3D canopy structure is explicitly described. These models require calculation of the distribution of light energy on the 3D plant structure, to integrate physiological processes from organ to plant level. This has motivated the development of new radiative models. We introduce first the principles governing the physical interactions between light and a plant canopy. We present then operational models. Fast methods have been developed for calculating sun and sky light directly intercepted by plant organs. Such methods may be used for the simulation of processes depending on the UV or PAR radiations. Models taking into account the multiple scattering between plant elements are based either on Monte Carlo ray tracing or on the radiosity method. We present the principle of these approaches and recent developments on their applications to crop modelling. (৫ Inra/Elsevier, Paris.)
\end{abstract}

\section{light microclimate / ray tracing / projection / radiosity / virtual plants}

Résumé - Modèles radiatifs pour la modélisation architecturale. Le microclimat lumineux détermine de nombreux aspects de la croissance des plantes. La prise en compte des interactions lumière-végétation dans un modèle de fonctionnement nécessite un modèle radiatif calculant les flux à l'échelle spatiale retenue pour décrire les processus biologiques. Les modèles architecturaux 3D décrivent les plantes fonctionnellement et géométriquement comme un ensemble d'organes interconnectés. La géométrie de chaque organe étant définie, la structure 3D du couvert végétal est décrite explicitement. Ces modèles nécessitent le calcul de la distribution de la lumière sur la structure 3D des plantes, afin d'intégrer les processus de l'organe à la plante. Cela a motivé le développement de nouveaux modèles radiatifs. Nous introduirons en premier lieu les principes généraux gouvernant l'interaction physique entre la lumière et le couvert végétal. Nous présenterons ensuite des modèles opérationnels. Des méthodes rapides ont été développées pour estimer la lumière en provenance du soleil ou du ciel directement interceptée par les organes végétaux.

Communicated by Gérard Guyot (Avignon, France)

* Correspondence and reprints

chelle@bcgn.grignon.inra.fr 
De telles méthodes peuvent être utilisées pour la simulation de processus biologiques dépendant de l'ultraviolet et du rayonnement photosynthétiquement actif. Les modèles prenant en compte les multiples diffusions entre les éléments de plante sont basés soit sur le lancer de rayons stochastique, soit sur la méthode de radiosité. Nous présenterons le principe des ces approches et les récents développements de leurs applications à la modélisation du fonctionnement des cultures. (@ Inra/Elsevier, Paris.)

\section{microclimat lumineux / lancer de rayons / projection / radiosité / plantes virtuelles}

\section{Introduction}

\subsection{Light and plant development}

Radiative transfer conditions many aspects of crop growth and development. Photosynthesis depends on the energy absorbed in the spectral domain of the photosynthetically active radiation PAR (400-700 nm). Photomorphogenesis is driven by photoreceptors such as phytochrome or cryptochrome. Phytochromes are reactive to the red $(660 \mathrm{~nm})$ and the far red $(730 \mathrm{~nm})$ radiations. Cryptochromes are reactive to the ultraviolet (UV) and the blue $(320-500 \mathrm{~nm})$. The light quality in the solar spectrum also determines the development of plant diseases, e.g. those caused by fungi [29]. Temperature also influences greatly crop growth and development. Radiative energy absorbed by an organ in the solar spectrum represents a large contribution to the global energy budget of the organ, which determines its temperature. Moreover, the PAR irradiance controls the opening of stomata, which regulates energy transfer. Conversely, plant development determines the radiative budget of the canopy through the canopy structure and the optical properties of organs.

\subsection{Upscaling from organ to canopy level}

Biological processes occur at the level of plant organ (leaf, bud, internode). Modeling the crop growth and development requires a scaling-up of the functioning of plant organs to the canopy level. This is performed in usual crop models by means of two simplifications: i) the functioning of a canopy is described by that of one 'mean plant', whose behaviour is supposed to represent the aver- age behaviour of the plants population; ii) the structure is described by statistical variables characteristic of the canopy level, e.g. the leaf area index (LAI), which is the quantity of leaf area per unit area of soil. Finally, this approach considers the canopy as a biophysic medium and does not take into account entities such as individual plants or organs. This approach has led to several efficient crop models (see Hanks and Ritchie [24] for a review), but does not allow a consideration of horizontal heterogeneity and plant to plant variability within the population.

An alternative approach consists in modeling the canopy as a population of interacting plants and a plant as a set of interacting organs $[28,39,42]$. It takes into account the duality of the plant organ, that is the physiological functions and the 3D geometry, which governs its exchanges with its physical environment. We will refer to such models as 'architectural models'; most of the models are based on the L-system theory [30]. Modeling the interactions between organs and their physical environment requires the exchange of information between the plant model and external models ( $f i g$ ure 1). These communications have been introduced at the level of individual plant organs in recent architectural models $[19,32]$

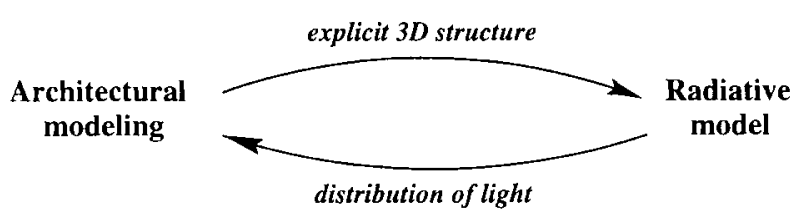

Figure 1. Interactions between an architectural model and a radiative model. 


\subsection{Radiative models for architectural model}

A radiative model adapted to the architectural approach must provide light fluxes received by each organ. Modeling the radiative exchanges within a canopy consists in the characterization of interactions (reflection, transmission, absorption) between light and organs, and the integration of these local processes over the whole structure. The complexity of this integration depends on the level of approximation in the structure description. We will now discuss the two approaches enabling this integration.

\subsubsection{TM approach}

The TM approach is based on the transport theory [8], which considers light propagation in a continuous medium composed of infinitely small scatterers. The spatial variability of the canopy structure is taken into account by dividing the canopy into volume elements and describing statistically the plant organs contained in this volume. A volume element can be a horizontal layer, a tube or a cell. Models based on this approach provide fluxes averaged over volume elements. They are efficient to estimate fluxes at the canopy scale and are intensively used with crop models based on the 'mean plant' approach. To be used for architectural modeling, their output, which is the field of fluxes averaged by volume element, must be down-scaled to the organ level. The fineness of spatial mesh is difficult to choose: cells should be small enough to describe the spatial variations of structure and fluxes, but large enough such that the transport theory can be applied [26]. The first models were not able to deal with the intra-cell variability of structure and fluxes. Some works introduced into the TM approach the spatial distribution of organs within a volume element $[26,34]$. These formalisms are complex and required parameters difficult to estimate. This is the case, for example, for the clumping parameter [35]. The intra-cell variability of fluxes may mainly be explained by the finite size of scatterers, which creates spatial discontinuities within the cell. Knyazikhin et al. [27] proposed a way of introducing finite-dimensional leaves into the TM approach. However, their method took these discontinuities into account only statistically and then refined only the mean value of fluxes. Anisimov and Fukshansky [2] introduced the statistical moments of the functions describing the structure within a cell. This enables an estimation of the variance associated with the mean fluxes calculated for each cell. This improved the estimation of fluxes compared to classic TM models [3]. However, the variability of fluxes within a cell is still described at the scale of the cell and not at the scale of an organ.

Moreover, the irradiance of a surface depends on the angular distribution of the incident light. The estimation of the irradiance of a surface from the output of TM models requires that the mean fluxes are described by a distribution of radiance. This is performed by only the most complex and time consuming TM models such as that of Myneny et al. [33] or DART [20].

TM models described statistically the radiative fluxes at the level of a volume element. Works cited above aimed at improving the estimation of these fluxes. However, a volume element should contain a large number of organs and high variability on the fluxes may exist between individual organs. This variability cannot be estimated by the TM approach, since the exact position and orientation of individual organs is not taken into account.

\subsubsection{Surface-based approach}

Different methods have been set to describe explicitly the 3D canopy structure as a set of geometric primitives: direct measurements by $3 \mathrm{D}$ digitizers [49], use of statistical distributions of parameters describing the shapes and positions of organs $[4,17,41]$ or simulation of plant growth $[19,32]$. Progress in computer science has now enabled the modeling of radiative transfer within a canopy structure described explicitly $[6,16,21]$. We will refer to these models as surface-based models. The advantages of this approach are to provide fluxes distributed on individual geometric primitives and to take into account the geometry of each foliage element (size, position, orientation). The stumbling block is the numerical complexity of the integration when the canopy structure is described by a large set of primitives. The level of 
detail of the geometry required to obtain an expected accuracy of radiative simulations is still being studied. For example, the undulation of maize leaf has a negligible influence on the interception of light within a canopy [18].

In this paper we will consider the estimation of radiative fluxes on individual organs. Thus, we will focus on the surface-based approach for the radiative modeling, including intermediate models that combine the TM and the surface-based approaches. In section 2, we present how light and organs interact within a canopy. Section 3 presents methods to estimate the amount of light coming directly from sun and sky to organs. Surface-based models enabling us to simulate high levels of multiple scattering are described in section 4 .

\section{Light and organ}

We will first describe a canopy as a radiative system and introduce the radiance equation, which expresses the radiative equilibrium at a point $P$ on an organ, at a time $t$ and for a wavelength $\lambda$. We will discuss the integration of this equation to estimate the radiative conditions driving biological processes. Finally we will introduce a classification of surface-based models based on the approach used to solve the radiance equation.

\subsection{The radiance equation}

In the solar spectrum, natural light sources are only the sun and the sky. Solar radiations intercepted by an organ may come directly from the sky hemisphere and after scattering from other organs. The distribution of direct light within the canopy depends on the structure of the canopy and on the angular distribution of radiance characterizing the sky hemisphere. The light intercepted by an organ is absorbed and scattered, that is reflected and transmitted, to other plant organs. The proportion between these two phenomena varies with the wavelength (figure 2). The angular distributions of the reflected (resp. transmitted) light are described by the bidirectional reflectance distribution func-

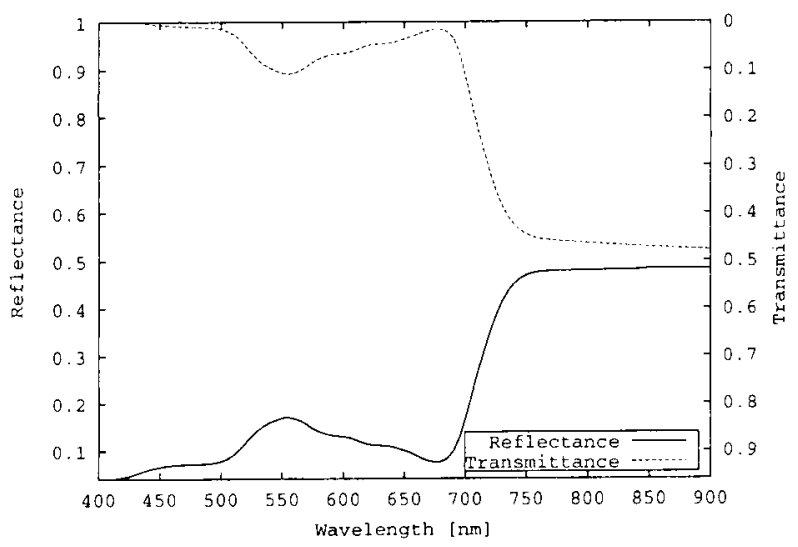

Figure 2. Spectrum of the optical properties (reflectance, transmittance) of a Walnut tree leaf measured with the spectroradiometer LI-COR LI-1800 (from D. Combes et al. [14]).

tion (BRDF) (figure 3) (resp. the bidirectional transmittance distribution function (BTDF)). The quantity of radiation scattered from a surface element $S_{i}$ and intercepted by another surface element $S_{j}$ depends on the BRDF or BTDF of $S_{j}$ and on the relative positions and orientations of $S_{i}$ and $S_{j}$. These relations are described by the radiance equation, which expresses the radiance scattered by a surface element $S_{i}$ at a point $P$ in a direction $\overrightarrow{\omega_{r}}$ as a function of the radiance incoming at the point $P$ from all the directions $\bar{\omega}$ (figure 4 ):

$$
L\left(P, \overrightarrow{\omega_{r}}\right)=L^{1}\left(P, \overrightarrow{\omega_{r}}\right)+L^{x}\left(P, \overrightarrow{\omega_{r}}\right)
$$

where $L^{\prime}\left(P, \overrightarrow{\omega_{r}}\right)$ and $L^{v}\left(P, \overrightarrow{\omega_{r}}\right)$ are, respectively, the contribution of direct and scattered light to the radiance scattered at $P$ :

$$
\begin{aligned}
& L^{1}\left(P, \overline{\omega_{r}}\right)=\int_{\overrightarrow{\omega_{s}} \cdot \vec{n}<0} f_{r}\left(\overline{\omega_{s}}, \overline{\omega_{r}}\right) L\left(P, \overline{\omega_{s}}\right) \cos \theta d \omega_{s} \\
& +\int_{\overline{\omega_{s}} \cdot \vec{n}>0} f_{t}\left(\overrightarrow{\omega_{s}}, \overrightarrow{\omega_{r}}\right) L\left(P, \overrightarrow{\omega_{s}}\right) \cos \theta d \omega_{s} \\
& L^{x}\left(P, \overrightarrow{\omega_{r}}\right)=\int_{\overline{\omega_{i} \cdot n}<0} f_{r}\left(\overrightarrow{\omega_{i}}, \overrightarrow{\omega_{r}}\right) L\left(P, \overrightarrow{\omega_{i}}\right) \cos \theta d \omega_{i} \\
& +\int_{\overrightarrow{\omega_{i} \cdot \vec{n}>0}} f_{t}\left(\overrightarrow{\omega_{i}}, \overrightarrow{\omega_{r}}\right) L\left(P, \overrightarrow{\omega_{i}}\right) \cos \theta d \omega_{i}
\end{aligned}
$$




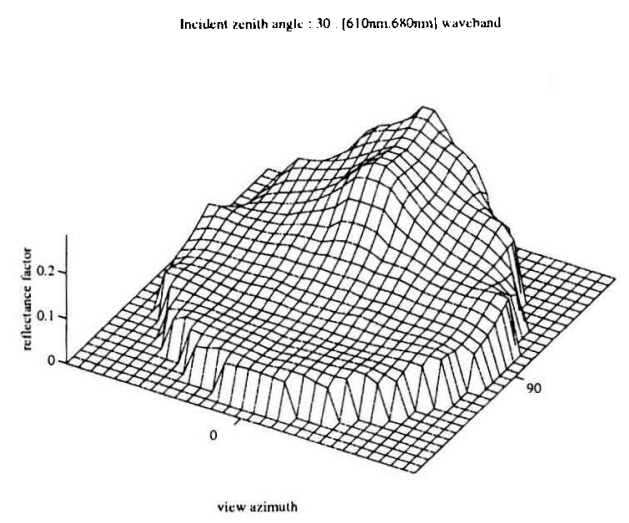

(a)

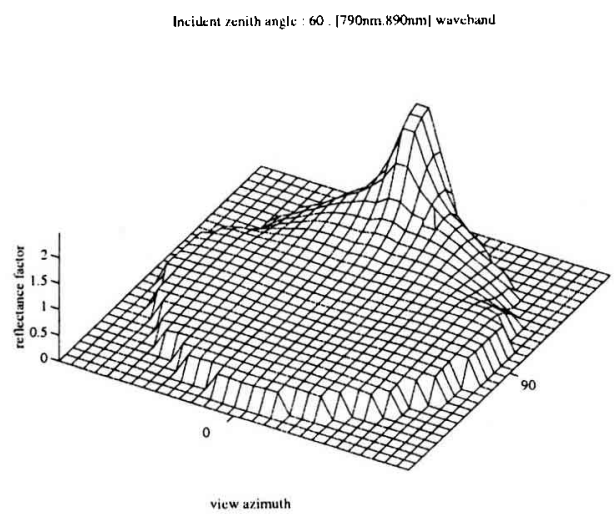

(b)

Figure 3. BRDF of a maize leaf for two wavelengths and two incidence angles of light (from Sanz et al. [45]). a) Red, 30 ; b) NIR, $60^{\circ}$.

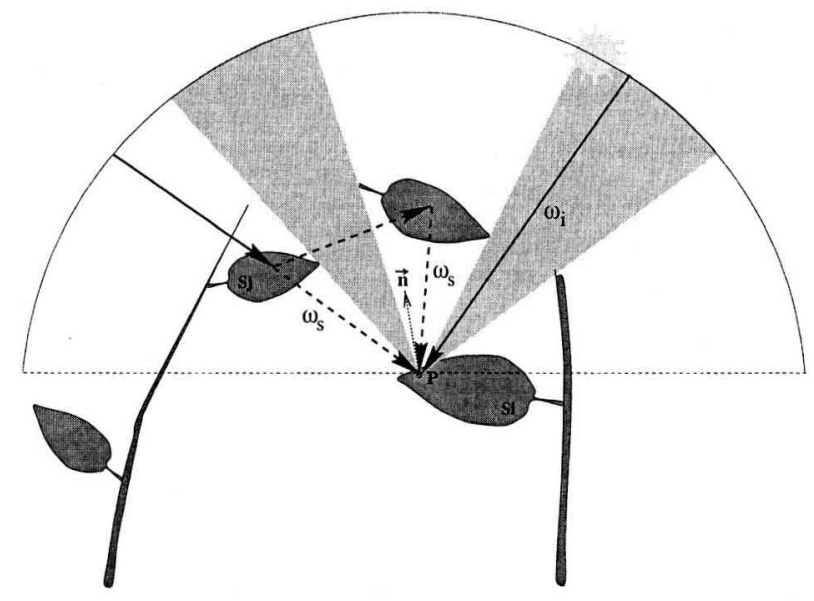

Figure 4. Radiative budget of a plant organ at a point $P$ (grey parts correspond to the solid angle domain through which $P$ is directly lit by sky and sun light; white parts to the solid angle domain through which $P$ is not directly lit).

where $\overrightarrow{\omega_{s}}$ belongs to the set of directions in which there is a free path between $P$ and the top of the canopy; $\overline{\omega_{i}}$ belongs to the set of directions in which there is not a free path; $d \omega$ is an elementary solid angle around the direction $\vec{\omega} ; \theta$ is the angle between $\vec{\omega}$ and the normal $\vec{n}$ to $S_{i}$ at point $P ; f_{r}$ and $f_{t}$ are respectively the BRDF and the BTDF of $S_{i}$ at the point $P$.
The solution of equation (1) is the field of radiance $L(P, \vec{\omega})$ over the set of surfaces describing the canopy, at a time $t$ and for the wavelength $\lambda$. $L(P, \vec{\omega})$ must be integrated over space, direction, time and wavelength. However, we will see below that for the simulation of biological processes, the integration scheme is connected to the biological response.

\subsection{Radiative variable for biological processes}

Photo-biological processes depend on the quantity of light intercepted by an organ, but also on the characteristics of the process in terms of space scale, temporal, angular and spectral sensitivity.

The organ response may be a non-linear function of light. This is the case of photosynthesis, for example. For such functions, $L(P, \vec{\omega})$ should be integrated over area and period, for which the variations of $L(P, \vec{\omega})$ are low. Regarding the spatial scale, high gradients of light over the organ surface may exist. For example, organs may have some parts shaded, in sun-flecks or in the penumbra. Penumbra is an area of transition between fulllit and shaded area that comes from the area of the 
sun. Sun course and the variation of cloudiness explain the temporal variations of the light coming from the sky hemisphere. Several phenomena generate both temporal and spatial variations of light. For example, the movements of vegetation modify both spatially and temporally the distribution of light.

Moreover, the response depends on the kind and the state of the organ. First, the response law may vary over the surface of the organ owing to variations in physiological parameters, such as the nitrogen content, or in physical parameters, such as the temperature. Second, the past of an organ may also modify its response. For example, photo-inhibition and photo-activation phenomena make the photosynthetic response of an organ depend on its radiative history. This has been shown in sun-flecks [36]. Third, the sensibility of biological photoreceptors to the direction of incoming light is not well established. Some studies showed that some leaf tissues such as the epidermis or palisade may act as optic systems such as a lens [38] or a tunnel [52].

A light-driven process has then a response function $g_{b}$ depending on the incident radiance $L(P, \vec{\omega})$ and on space, time, light direction and wavelength. The radiative parameter $R_{b}$ of such a process could be defined by:

$$
R_{b}(S, \Delta t, \Delta \lambda)=\int_{S} \int_{\Delta} \int_{\Delta \lambda} \int_{\omega} g_{b}\left(L_{t, \lambda}(P, \vec{\omega}), P, t, \lambda, \vec{\omega}\right) d \omega d \lambda d t d S
$$

The estimation of $R_{b}$ should be performed by dividing the space of integration in intervals, where $g_{b}$ nearly is a constant or linear function of light. This simplifies the calculations so:

$$
R_{b}(S, \Delta t, \Delta \lambda)-g_{b}\left(\int_{S} \int_{\Delta t} \int_{\Delta \lambda} \int_{\omega} L_{t, \lambda}(P, \vec{\omega}) d \omega d \lambda d t d S, P, t, \lambda, \vec{\omega}\right)
$$

Taking into account the spatial and temporal variations having a high frequency, such as those due to the movements of plants and clouds or the sun-fleck phenomena, would require a model of biological processes with short time step, suborgan scale and narrow spectral band and an improvement of the knowledge of the biological response at these high frequencies. The effects of high spatial and temporal frequencies are then often neglected for agronomical applications. The space and time scales are chosen such that the variations of the canopy structure and of the lighting conditions are low. Thus, agronomical models usually have a time step from $1 \mathrm{~h}$ to a 1 day, a spatial scale of an organ or plant and a small number of spectral band: PAR, NIR and the whole spectrum.

\subsection{Simplifications of the radiance equation}

A surface-based model adapted to architectural modeling shall solve the radiance equation for several intervals of space, time and wavelength. Due to the complexity of this equation, simplifications have to be applied to generate efficient but restricted models.

The number of organs for which radiative parameters must be estimated depends on the biological model. For example, photosynthesis implies all the green leaves, whereas some photomorphogenetic models focus on light microclimate at the level of apices or internodes. This led to two schemes of radiative modeling: the global and local approaches. In the global approach, the radiative variables are estimated for all organs. In the local approach, the radiative variables are estimated on a restricted number of organs, which reduces the number of calculations.

The complexity of the radiance equation comes mainly from the calculation of multiple scattering. The weight of multiple scattering in the incoming light on organs varies spectrally (figure 2). Two spectral domains may be defined: the UV and PAR where multiple scattering is low and the infrared where it is high. Estimating fluxes in the UV and PAR domains can be highly simplified, if multiple scattering is neglected. In the infrared, the radiance equation must be solved, which necessites complex models. We will now present how direct light and multiple scattering are calculated with the surfacebased approach. 


\section{Direct light}

\subsection{Calculation of direct light}

\subsubsection{Principle}

Calculating the direct lighting of a set of surfaces requires to sample directionally the sky hemisphere and spatially the area of the surfaces $x$ and to determine for each element $(P, \vec{\omega})$ within the sample, if there is a direct path from the point $P$ to the top of canopy in the direction $\vec{\omega}$. In the global approach, these samplings are performed by following the propagation of light from sky and determining the surface element hit by each light ray. For the local approach, the sampling is performed by following the inverse sense of light propagation, from the surface elements of interest to the sky hemisphere.

The sampling of the sky hemisphere relies usually on a discretization in solid angle elements $\Delta \omega$, such that the radiance within $\Delta \omega$ is assumed constant. This amounts to approximate the continuous sky hemisphere as a finite set of punctual collimated light sources. Because the dimensions of the canopy are small compared to the distance to the light sources, any extended light source can be approximated as a set of punctual light sources, so that the approach is very general.

The irradiance of a surface is the sum of the contribution of each individual source; thus, we will focus on the calculation of direct light from a single source. The irradiance of a surface $S_{i}$ by light coming from a single direction is proportional to the apparent surface $S_{i}^{*}$, that is the surface that would be seen by an observer looking at the canopy from the position of the light source. The apparent surface depends on the orientation of $S_{i}$ relative to $\vec{\omega}$ and on the possible shading due to other surfaces. We describe below how it can be estimated by ray casting or by projection.

\subsubsection{Ray casting}

In the global approach, this method consists in casting several rays from the light source to the canopy. Rays are cast in the direction from points sampled on a plane above the canopy. The sampling of the plane may be performed regularly or stochastically. The propagation of a ray stops when it intersects a primitive (figure $5 a$ ). $S_{i}^{*}$ is then proportional to the number of rays caught by $S_{i}$. The drawback of this method is the high number of rays required to reach a satisfying accuracy.

For the local approach, rays are cast from points randomly chosen on the surfaces of the organs of interest in the direction $\vec{\omega} . S_{i}^{*}$ is then proportional to the number of rays starting from $S_{i}$, which exit the canopy (figure $6 a$ ).

\subsubsection{Projection}

The second method for the global approach consists in projecting in parallel the primitive on a discretized screen located above the canopy and normal to the direction of light (figure $5 b$ ). Shaded primitives are determined by applying the Z-buffer algorithm, which consists to update of a pixel of the discretized screen with the identifier of the closest primitive to the screen. Thus, $S_{i}^{*}$ is proportional to the number of pixels covered by the projection of $S_{i}$. This method is very efficient in terms of speed and accuracy.

In the local approach, the irradiance due to the whole sky hemisphere is generally calculated successively for each $S_{i}$. A hemispherical projection centered on a point $P$ of $S_{i}$ provides an image, where each pixel corresponds to a solid angle (figure $6 b$ ). An empty pixel corresponds to a direction in which the sky lightens the point $P$. The irradiance in $P$ is calculated by summing the irradiances due to the sky radiances associated with each empty pixel. To obtain the irradiance of $S_{i}$, the method is applied for different points $P$ over $S_{i}$.

\subsection{Applications}

\subsubsection{PAR irradiance}

In the PAR spectral domain, the contribution of multiple scattering to leaf irradiance is often neglected. We have analysed this approximation with a Monte Carlo ray tracing [10]. We considered a canopy of 20 digitized maize plants at mature stage with a LAI of 4.9. The canopy was 


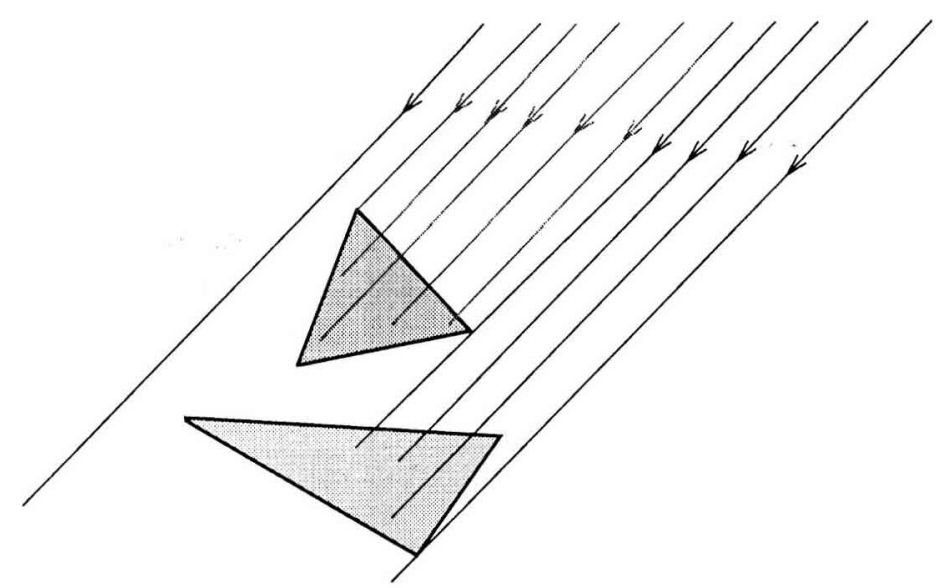

(a)

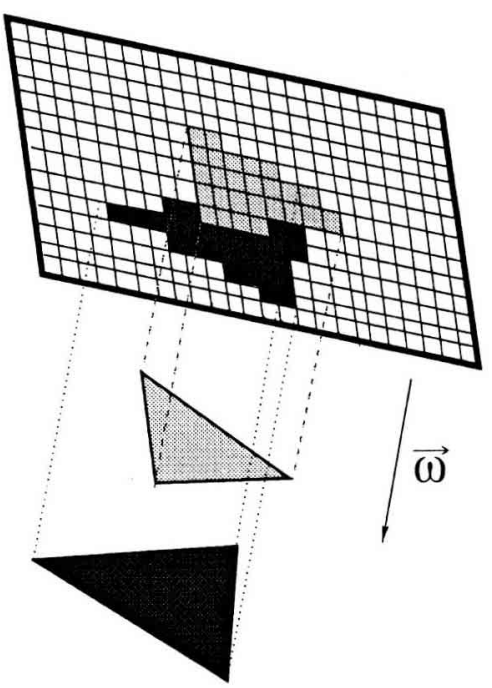

(b)

Figure 5. Calculation of the interception of direct light by two surfaces with the global approach: a) by ray casting; b) by Z-buffer.

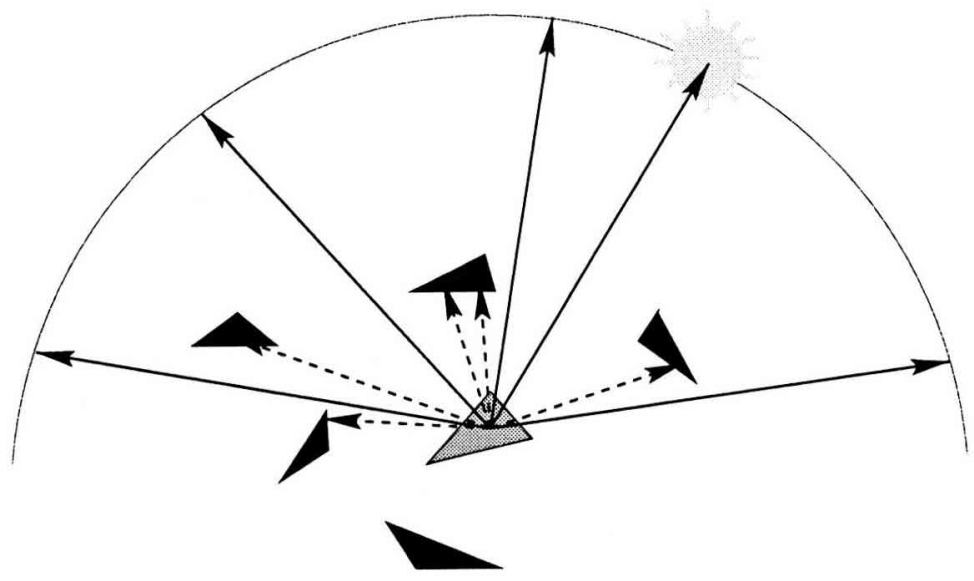

(a)

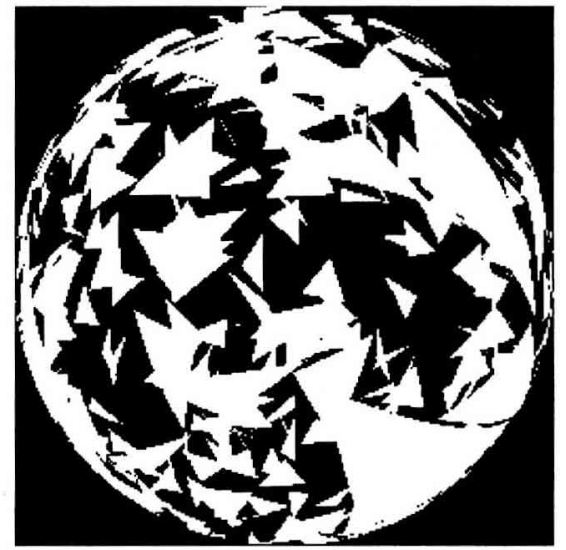

(b)

Figure 6. Calculation of the interception of direct light by a surface with the local approach: a) by ray casting; b) by hemispherical projection.

illuminated by a light source at the zenith in the PAR domain [11]. Hemispherical optical properties were used for this simulation and the two faces of a leaf had the same properties. Reflectance was 0.10 for leaves and stems and 0.15 for soil. The leaf transmittance was set at 0.06 . The proportion of multiple scattering in the energy absorbed by all leaves is only $3.8 \%$. Thus neglecting multiple scat- tering in the PAR domain seems a satisfying compromise for many agronomical applications.

As seen in the previous sub-section, the most efficient method to estimate direct light is projection. Dauzat [15] used this method to simulate fisheye views of forest and the light pattern at the ground level beneath an oil palm stand. The projec- 
tion method has also been applied to estimate the irradiance of organs in the PAR in a crop model of maize [19] and to estimate the interception of light by a coffee tree to model water fluxes [40].

\subsubsection{Study of the canopy structure}

Interception of direct light depends only on the structure of the canopy, and thus can be used as an indirect measurement of it. The methods presented above have been used to validate the structure of computer representations of plants and to study structural parameters difficult to measure in the field.

The gap frequency at an elevation $z$ in a direction $\vec{\omega}$ is the probability for a light ray following $\vec{\omega}$ to reach the elevation $z$ within a canopy without interception. This variable can be estimated on virtual canopies by projection or ray casting and is widely used in the field to characterize actual canopies, e.g. using hemispherical photographs. The comparison of gap frequencies measured in field and calculated by projection has been used to validate the structure of maize mock-ups generated by various methods $[1,18,19]$.

In the TM approach, the distribution of leaf position within a volume element is often assumed to be random. This enables using the Beer-Lambert law to calculate the interception of direct light. To deal with non-random foliage distributions, Nilson [35] introduced an additional parameter in the Beer law, the clumping parameter $\lambda_{0}$. The case of random leaf position corresponds to $\lambda_{0}=1$, whereas $\lambda_{0}<1$ corresponds to clumping and $\lambda_{0}>1$ corresponds to shade avoidance. The use of the clumping parameter is, however, restricted because it is difficult to measure in the field. This parameter may be estimated from calculation of interception on computer mock-ups of canopies [9, 18]. Figure $7 a$ shows the variation of $\lambda_{0}$ as a function of the position of the sun for a virtual maize canopy. Figure $7 b$ compares the interception of light computed following the Beer-Lambert law and by the projection method. This allows us to know in which cases Beer-Lambert-based models provide correct estimations of light interception.
The distribution of foliage has been also described by fractal parameters in the case of forests. Knyazikhin et al. [26] used computer mock-ups to estimate the fractal dimension of the crown volume of Norway spruce and proposed an improvement of the Beer law in the case of fractal structure.

\section{Modeling multiple scattering of light}

\subsection{Solving the radiance equation}

Estimating multiple scattering requires the solution of the radiance equation (equation (1)). As this equation is set at one point, an integration over all surfaces is required. Two approaches enable this integration: the stochastic ray tracing and a deterministic method derived from the finite elements: the radiosity method.

\subsubsection{Monte Carlo ray tracing}

The stochastic ray tracing relies on the Monte Carlo method [25]. It consists in calculating successively the path and interactions with surfaces of a large number of photons, until they exit the canopy or they are absorbed by surfaces (figure 8a). Global and local approaches were developed: rays are traced from the light sources to the canopy and from the organs of interest to the light sources, respectively. The Monte Carlo approach is very general and requires few assumptions. Thus, Monte Carlo models enable simulations for nearly any type of light sources, canopy structure or optical properties of organs. It enables us to simulate a large set of variables, such as fluxes over individual organs, canopy BRDF or radiance distribution at different levels within the canopy. Moreover, it enables us to separate the contribution of the different orders of scattering to the radiative variables. On the other hand, results are statistical estimates of mean values. The variance associated with an estimated variable depends on the number of photons that contribute to this result. This number may be low for very small or weakly illuminated surfaces. In these cases, obtain- 


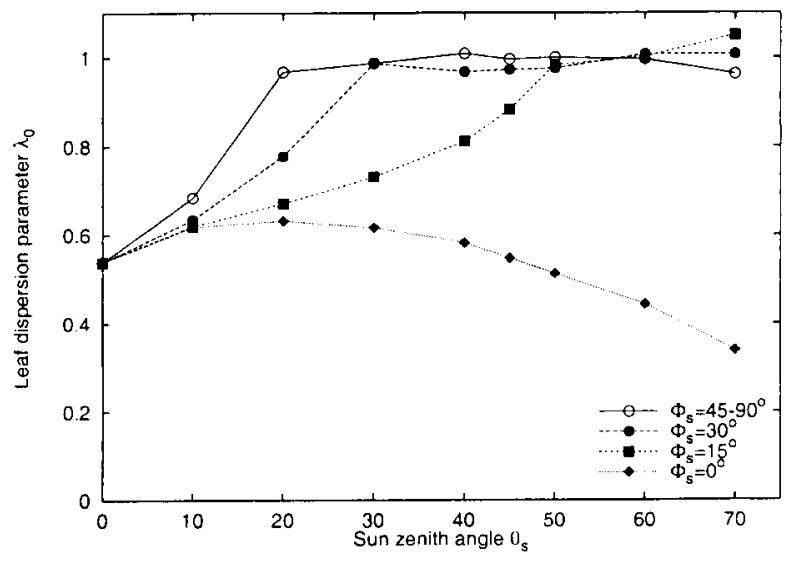

(a)

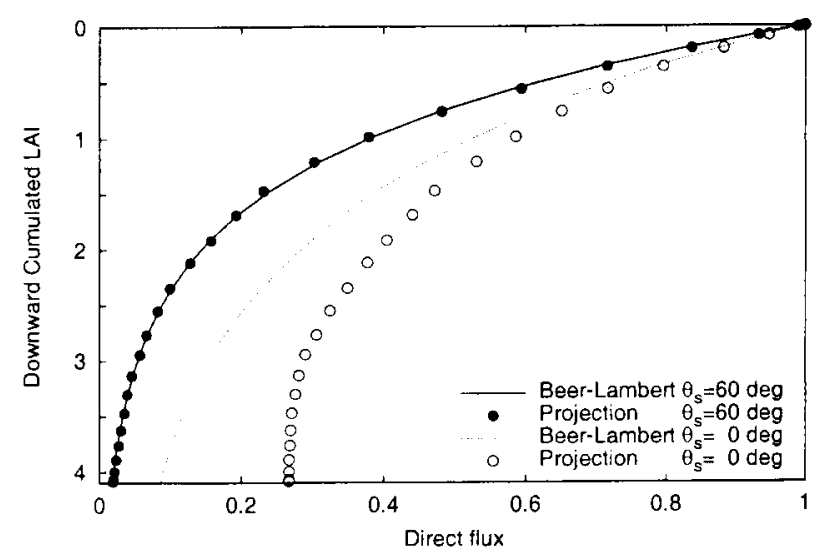

(b)

Figure 7. Effect of leaf dispersion on the light interception by a full-developed maize canopy. a) Angular dependence of $\lambda_{0}\left(\Phi_{\mathrm{s}}\right.$ is the sun azimuth angle); b) profile of direct flux for two positions of the sun.

ing low variances for such surfaces requires us to either privilege these surfaces by using the local approach or trace a large number of rays in the case of the global approach. The variance on each result can be estimated at the end of a simulation [10]. To reach an expected accuracy, the simulation can be performed by iterations, variances being estimated at the end of each step. Obtaining a correct accuracy for a large number of organs then requires very large simulation times on usual workstations. The Monte Carlo approach enables us in principle to deal with any kind of BRDF. However, taking into account anisotropic BRDF often requires using time-consuming procedures to sample the direction of scattering. Moreover, it requires an accurate description of the 3D shape of leaves to avoid numerical artefacts. Finally, due to the computational complexity, the case of anisotropic BRDF has seldom been addressed in radiative simulations on plant canopies.

Owing to these features, the Monte Carlo method is generally used as a tool to investigate the radiative behaviour of canopy or as a reference to evaluate simpler models. But it is too slow to enable the coupling with a crop growth model.

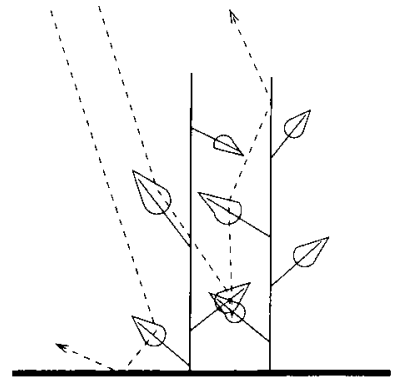

(a)

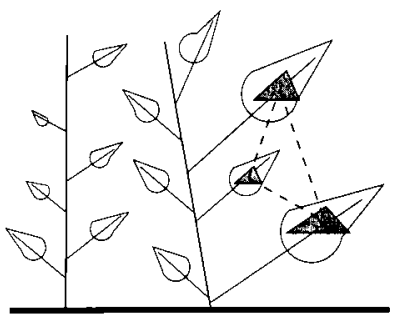

(b)
Figure 8. Principle of surface-based methods to simulate radiative exchanges within canopies: a) Monte Carlo ray tracing; b) radiosity.

\subsubsection{Radiosity-based methods}

The radiosity method $[22,50]$ is based on the assumptions that surfaces are lambertian and that radiative fluxes over a primitive are constant. This enables the approximation of the radiance equation as a system of linear equations. The radiosity equation expresses the radiant exitance or radiosity $\mathrm{B}_{i}$ over a surface $S_{i}$ as the reflection and the transmission of the incoming light (figure $8 b$ ). This incoming light is expressed as a linear combination of the 
radiosities $B_{j}$ of the other surfaces. The coefficients of this linear combination are called form factors and represent the proportion of energy scattered by a surface $S_{j}$ that reaches a surface $S_{i}$. A form factor between $S_{j}$ and $S_{i}$ is calculated by an integration over the two surfaces. If there is no surfaces between them (figure $9 a$ ), a standard numerical integration can be performed. Moreover, if $S_{i}$ and $S_{j}$ are polygons, an analytical formulation of the form factor has been established [46]. When there are occlusions, the really exchanging part of $S_{i}$ and $S_{j}$ is to be determined and calculations are more complex (figure 9b). These calculations are usually simplified by calculating the form factor between $S_{i}$ and an elementary surface located at the center of $S_{j}$. This method is called the method of pointsurface form factor. This approximation is incorrect for some geometrical configurations [5], mainly when the two surfaces are very close.

No local approach was developed for radiosity: models use a global scheme. The first radiosity models consisted in computing the form factors between each pair of surfaces $\left(S_{i}, S_{j}\right)$ and solving the resulting system. The requirement in memory storage and in simulation time was proportional to $o\left(N^{2}\right)$, where $N$ is the number of primitives describing the 3D structure. This limited the use of such models to simple structures. Many works in computer graphics improved the ability of this method to deal with large sets of primitives (see Cohen and Wallace [13] for a review). These optimizations were developed for scenes within buildings. It is

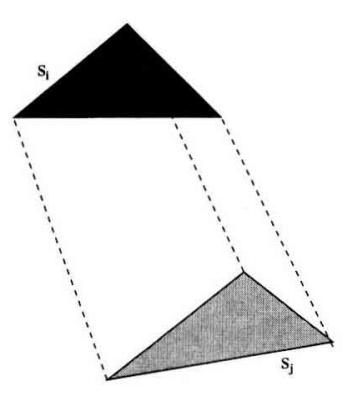

(a)

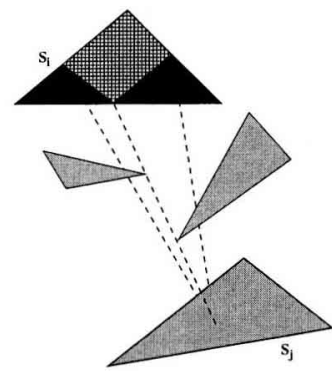

(b)
Figure 9. Geometry of the form factor calculation: a) without occlusion; b) with occlusions. difficult to apply these methods to plant canopies, whose optical and geometrical characteristics differ from that of building scenes: i) the spatial distribution of surfaces is broader in a vegetation canopy than in a room; ii) the contribution of high order of scattering is important in the near infrared for vegetation canopies, which makes inefficient the iterative schemes developed for building scenes. Other researches in computer graphics extended the radiosity method to non-lambertian surfaces [ 12 , 47, 48]. However, these methods seem at this time to be too complex to be applied to the vegetation.

Considering that the radiosity method is welladapted to compute the distribution of light over a small set of surfaces and that TM models estimate satisfactorily the spatial distribution of mean fluxes, we developed the nested radiosity [9] for crop cultures. Irradiance of a surface $S_{i}$ due to multiple scattering is calculated in two parts. First, the energy coming from all the organs far from $S_{i}$ is estimated globally from the field of mean fluxes provided by the SAIL model [51]. Second, the contribution of close organs is calculated by the radiosity method. The partition between close and far surfaces from $S_{i}$ is realized by a sphere centered on $S_{i}$. The sphere diameter is chosen by the user.

\subsection{Applications}

Surface-based models can be coupled with an architectural crop model to simulate dynamically the interactions between light and plants in the solar spectrum. They are also useful for improving the knowledge on radiative transfer within a canopy.

\subsubsection{Modelling light for plant models}

Previous works simplified the Monte Carlo ray tracing to obtain reasonable simulation times. For forest simulations, simplifications consist in describing some parts of the tree structure such as crown or shoots as a volume, in which foliage is described as a turbid medium [7, 37]. Mĕch [31] developed a model coupled with the L-system tool $c p f g$. His model simulated the red/far red ratio of the energy absorbed by apices to simulate the 
development of a population of clonal plants. As photomorphogenetic processes were taken into account only at apices, he developed its stochastic ray tracing following the local approach.

Goel et al. [21] developed an efficient radiosity model for crop cultures, introducing a new method to compute form factors. However, due to the complexity in $o\left(N^{2}\right)$, their model was limited to a small number of plants (nine maize plants described by 3136 triangles).

The nested radiosity model is an interesting compromise between the accuracy and the efficiency required for the coupling with architectural model. The choice of the sphere diameter $D$, depends on the canopy structure, the level of multiple scattering and the expected accuracy. Figure 10 plots the root mean square error (rmse) on the exitance of all the surfaces calculated by nested radiosity as a function of the sphere diameter. Monte Carlo ray tracing was used as a reference. Simulations were performed for three stages of development of a maize canopy. This maize crop had a density of 10 plants. $m^{-2}$ and the distance between rows was $0.8 \mathrm{~m}$. The rmse decreased rapidly when $D_{s}$ was increased from 0 to $0.5 \mathrm{~m}$. For $D_{s}$ larger than $0.5 \mathrm{~m}$, the rmse remained nearly constant. Using $D_{s}=0.5 \mathrm{~m}$, a simulation on a canopy of 100 maize plants described with 56000 diffusers required $25 \mathrm{~min}$ on a Sun Ultra Enterprise (250 Mhz). Similarly to the classic radiosity, the nested radiosity enables us to dissociate the geometry-dependent calculations and the radiative calculations. Once form factors were computed and stored, the following simulations on the 100 maize plants took about 1 min. This makes the nested radiosity an efficient tool to study how the light climate within a given structure is affected by the characteristics of light source and the optical properties of leaves and soil. Figure 11 illustrates the use of this model to estimate the red/far red ratio distributed on plant organs.

\subsubsection{Towards a better knowledge of the radiative properties of a canopy}

Simulating radiative transfer with an explicit description of canopy structure should enable us to better understand the interactions between struc-

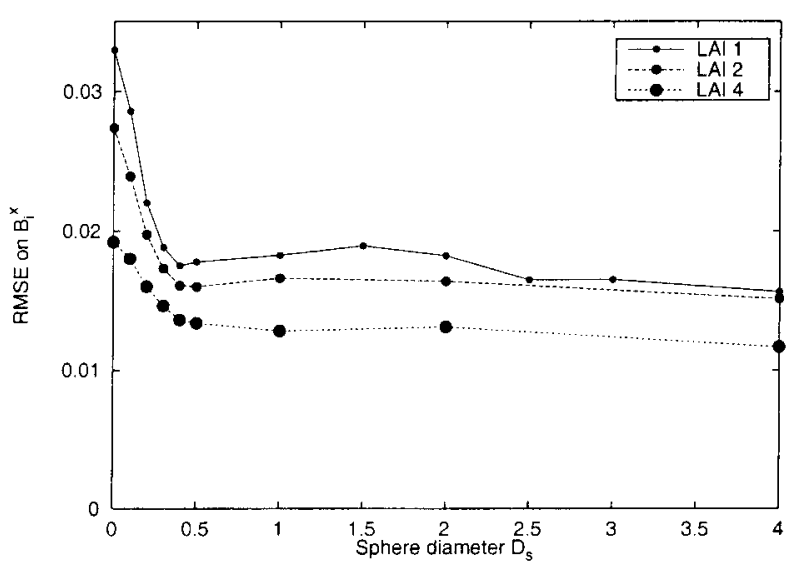

Figure 10. Influence of the sphere diameter on the rmse on the exitances calculated by the nested radiosity for virtual maize canopies at three stages

ture and radiation. This may be useful to improve the accuracy of TM models or to develope simplified models. For this kind of studies, the Monte Carlo ray tracing is well suited $[10,16,23,44]$.

To illustrate this field of application, we will present briefly a study of the directionality of scattered fluxes (see Chelle et al. [10] for a detailed presentation). In the TM approach, scattered fluxes are generally assumed to be isotropic. We investigated the validity of this assumption for a homogeneous canopy with lambertian leaves, using Monte Carlo ray tracing. Figure 12 shows the angular distribution of radiance for downward scattered fluxes at three elevations in the canopy. To compare these distributions, radiances for each elevation are normalized by the radiance in the vertical direction for the same elevation.

Close to the top of the canopy, fluxes were highly anisotropic. In contrast, they were close to isotropic lower in the canopy. As a general rule, the directional variation of scattered fluxes at a given level in the canopy nearly followed the directional probability of ray interception from that level to the top of the canopy. This property could be used to improve calculation of multiple scattering in the TM approach. It also implies that scattered fluxes are preferentially horizontal in the early stages of canopy development. This point may be important 


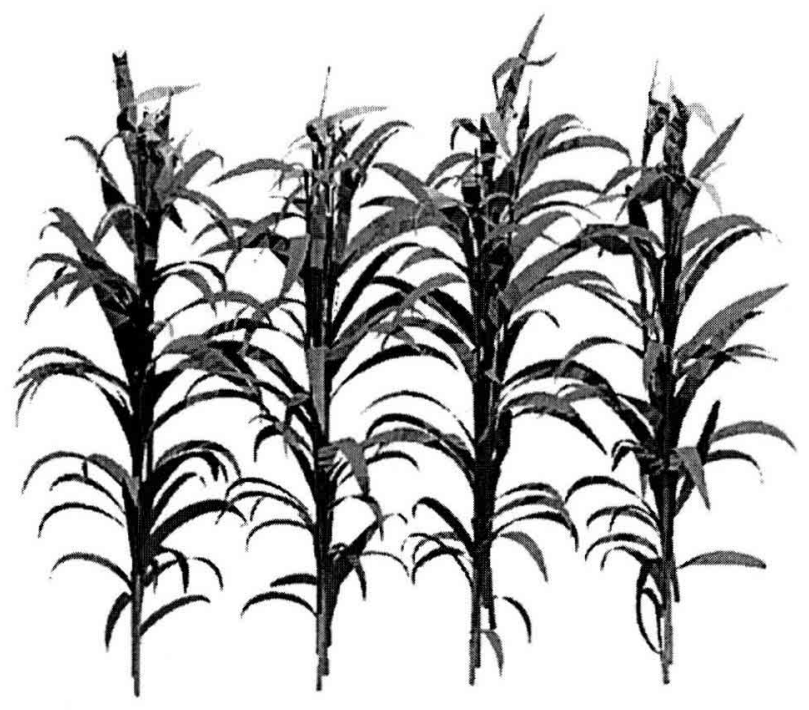

Figure 11. Distribution of the red/far red ratio on a maize canopy (the grey level is proportional to this ratio).

to take into account for simulating photomorphological processes occurring on vertical structure such as plant stems.

\section{Conclusion and future directions}

The development of 3D architectural models of plants has motivated the development of a surfacebased model for light: the 3D plant models provide an explicit description of the canopy geometry and they require the distribution of light energy on each plant organ.

To study biological processes sensitive to PAR and UV, multiple scattering may be neglected allowing fast calculations even on large 3D structures. The simulation of processes depending on infrared radiation is more complex. Radiositybased methods appear convenient, especially because of the dissociation of geometric and radiative calculations. However, the importance of neglecting specular reflection should be validated for agronomical applications. Moreover, the pointsurface method is not appropriate to calculate form factors when surfaces are very close. This makes it

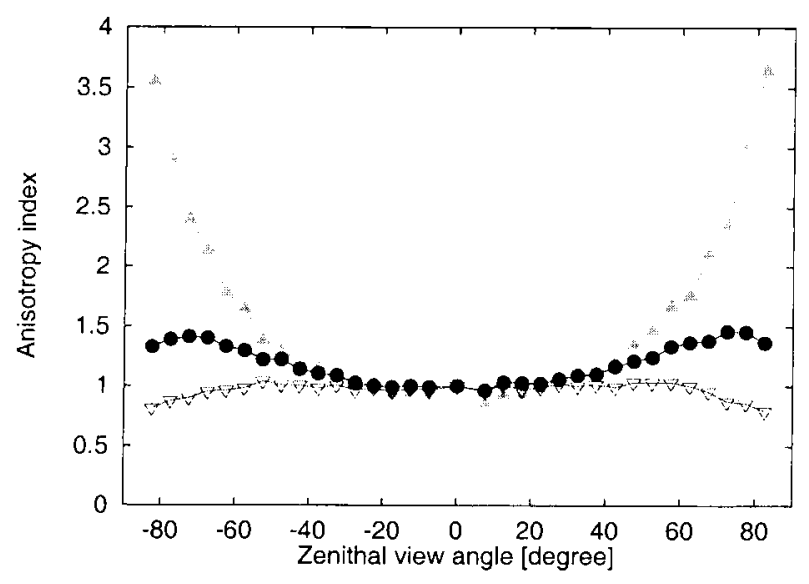

Figure 12. Directional variations of downward fluxes (scattering order $>1$ ) at three elevations expressed as the LAI above the considered level: $(\boldsymbol{\Delta}) \mathrm{LAI}=0.5,(\bullet) \mathrm{LAI}=2$ and $(\nabla)$ $\mathrm{LAI}=3.7$.

difficult to estimate light conditions in some specific plant parts such as the whorl of graminaceous.

Another important physical parameter of organ functioning is the temperature. The radiosity method could be coupled with models estimating sensitive and latent fluxes, in order to estimate the temperature of individual plants organs. However, this requires us to also take into account the biological regulations of the exchange properties of the surface, e.g. the stomatal control.

The plant growth and development are also studied indoors, in growth chambers or greenhouses. Modeling light within these environments differs from the case of the canopy in several aspects: there are radiative interactions between walls (large surfaces) and plants (set of small surfaces), the number of plants is small and then there is a high spatial heterogeneity and light sources. Owing to these features, the TM approach does not seem to be adapted to this environment. The complementarity between computer graphics model adapted to building scenes and canopy models seems to be an interesting starting point to develop a model for indoor canopies. 
Finally, a surface-based model taking into account the exact geometry of the canopy has also been used for remote sensing studies [6, 23, 43]. These models could improve the knowledge on the bound between radiative and structural properties of a canopy and signals acquired by a satellite. That would enable a better use of spatial data in crop studies.

To conclude, the three approaches to model radiative transfer within canopies are complementary. i) TM models efficiently estimate spatially averaged fluxes. They are well suited for applications where the canopy is dealt with as an entity, such as crop models based on the 'mean plant' concept or for remote sensing applications. ii) The Monte Carlo ray tracing appears to be a powerful tool to investigate the radiative properties of a canopy. iii) Projection and radiosity methods seem to offer the basis to develop operational models to couple with architectural models of plants. Moreover, intermediate approaches combining TM and surface-based models appear to be a promising direction to develop accurate and efficient radiative models for vegetation canopy. Beside these new possibilities opened up by surface-based models, research is still needed to make it possible to address the variability of light microclimate when high spatial or time resolution are considered.

\section{References}

[1] Andrieu B., Ivanov N., Boissard P., Simulation of light interception from a maize canopy model constructed by stereo plotting, Agric. For. Meteorol. 75 (1995) 103-119.

[2] Anisimov O., Fukshansky L., Light-vegetation interaction: a new stochastic approach for description and classification, Agric. For. Meteorol. 66 (1993) 93-110.

[3] Anisimov O., Fukshansky L., Optics of vegetation: implications for the radiation balance and photosynthetic performance, Agric. For. Meteorol. 85 (1997) 33-49.

[4] Aries F., Prévot L., Monestiez P., Geometrical canopy modelling in radiation simulation studies, in: Varlet-Granchet C., Bonhomme R., Sinoquet H. (Eds.),
Crop Structure and Light Microclimate, Characterization and Applications, Inra Éditions, Paris, 1993, pp. 159-173.

[5] Baum D.R., Rushmeier H.E., Winget J.M., Improving radiosity solutions through the use of analytically determined form-factors, Comput. Graphics (SIGGRAPH '89 Proceedings) 23 (1989) 325-334.

[6] Borel C., Gerstl S., Powers B., The radiosity method in optical remote sensing of structured 3D surfaces, Remote Sens. Environ. 36 (1991) 13-44.

[7] Brunner A., A light model for spatially explicit forest stand models, For. Ecol. Manag. 107 (1998) $19-46$.

[8] Chandrasekhar S., Radiative Transfer, Clarendon Press, Oxford, UK, 1950.

[9] Chelle M., Andrieu B., The nested radiosity model for the distribution of light within plant canopies, Ecol. Model. 111 (1998) 75-91.

[10] Chelle M., Andrieu B., Fournier C., Analyse des échanges radiatifs au sein d'un couvert végétal par lancer de rayons stochastique: réflectance bidirectionnelle et micro-climat lumineux dans le couvert, in: Guyot G., Phulpin T. (Eds.), Physical Measurements and Signatures in Remote Sensing, vol. 1, Balkema, 1997, pp. 193-199.

[11] Chelle M., Drouet J.L., Using a measured 3D canopy architecture to estimate the repartition of light between plant organs, in: Stabilising Processes in Mixed Plant Communities, Society of Experimental Biology, J. Exp. Bot. 49 (1998) 42.

[12] Christensen P.H., Stollnitz E.J., Salesin D.H., DeRose T.D., Wavelet radiance, in: Fifth Eurographics Workshop on Rendering, Darmstadt, Germany, 1994, pp. 287-302.

[13] Cohen M.F., Wallace J.R., Radiosity and Realistic Image Synthesis, Academic Press Professional, Boston, 1993.

[14] Combes D., Sinoquet H., Varlet-Grancher C., Simulation of the spatial distribution of the morphogenetically active radiation (MAR) within an isolated tree canopy, Ann. Sci. For. (1999) in press.

[15] Dauzat J., Simulated plants and radiative tranfer simulations, in: Varlet-Granchet C., Bonhomme R., Sinoquet H. (Eds.), Crop Structure and Light Microclimate, Characterization and Applications, Inra Éditions, Paris, 1993, pp. 271-278.

[16] Dauzat J., Hautecur O., Simulation des transferts radiatifs sur maquettes informatiques de couverts végétaux, in: Proceedings of the 5th International 
Colloquium on Physical Measurements and Signatures in Remote Sensing, 1991, pp. 415-418

[17] España M., Baret F., Chelle M., Aries F., Andrieu B., A dynamic model of maize 3D architecture: application to the parametrisation of the clumpiness of the canopy, Agronomie 18 (1998) 69-627.

[18] España M., Baret F., Aries F., Andrieu B., Sensitivity of radiative transfer variables calculation to the accuracy of canopy structure description: the case of maize canopy as described by a 3D architecture model, Agronomie 19 (1999) (in this issue).

[19] Fournier C., Andrieu B., A 3D architectural and process-based model of maize development, Ann. Bot. 81 (1998) 233-250.

[20] Gastellu-Etchegorry J.P., Desmarez V., Pinel V., Zagolski $F$., Modelling radiative transfer in heterogeneous 3-D vegetation canopies, Remote Sens. Environ. 58 (1996) 131-156.

[21] Goel N., Knox S., Norman J., From artificial life to real life: computer simulation of plant growth, Int. J. Gen. Syst. 18 (1991) 291-319.

[22] Goral C.M., Torrance K.E., Greenberg D.P., Battaile B., Modelling the interaction of light between diffuse surfaces, Comput. Graphics (SIGGRAPH ' 84 Proceedings) 18 (1984) 212-22.

[23] Govaerts Y., Verstraete M., Evaluation of the capabiliy of BRDF models to retrieve structural information on the observed target as described by threedimensional ray tracing code, in: Multispectral Sensing of Forestry and Natural Resources, EUROPTO Proc., no. 2314-24, Rome, Italy, 1994.

[24] Hanks J., Ritchie J., Modelling plant and soil systems, no. 31 in Agronomy, ASA Inc., CSSA Inc., SSSA Inc., 1991.

[25] Kalos M.H., Whitlock P.A., Monte Carlo Methods, Vol. I, John Wiley \& Sons, New York, USA, 1986.

[26] Knyazikhin Y., Kranigk J., Myneni R., Panfyorov O., Gravenhorst G., Influence of small-scale structure on radiative transfer and photosynthesis in vegetation canopies, J. Geo. Res. 103 (1998) 6133-6144.

[27] Knyazikhin Y., Marshak A., Myneni R., Interaction of photons in a canopy of finite-dimensional leaves, Remote Sens. Environ. 39 (1992) 61-74.

[28] Kurth W., Morphological models of plant growth: Possibilities and ecological relevance, Ecol. Model. 75-76 (1994) 299-308.
[29] Leach C., Anderson A., Radiation quality and plant diseases, in: Barfield B.. Thomason I. (Eds.), Biometeorology in Integrated Pest Management, Academic Press, New York, USA, 1982, pp. 267-306.

[30] Lindenmayer A., Mathematical models for cellular interaction in development, part I and II, J. Theor. Biol. 18 (1968) 455-484.

[31] Měch R., Modelling and simulation of the interaction of plants with the environment using L-systems and their extensions, Ph.D. thesis, University of Calgary, November, 1997.

[32] Měch R., Prusinkiewicz P., Visual models of plants interacting with their environment, in: Proceedings of SIGGRAPH '96 (New Orleans), 4-9 August, 1996, pp. 397-410.

[33] Myneni R.B., Marshak A.K., Knyazikhin Y., Asrar G., Discrete ordinates method for photon transport in leaf canopies, in: Myneni R., Ross J. (Eds.), Photon-vegetation Interactions - Applications in Optical Remote Sensing and Plant Ecology, Springer-Verlag, 1991, pp. 45-109.

[34] Myneni R., Maggion S., Iaquinta J., Privette J., Gobron N., Pinty B., Kimes D.S., Verstraete M.M., Williams D.L., Optical remote sensing of vegetation: modelling, caveats and algorithms, Remote Sens. Environ. 51 (1995) 169-188.

[35] Nilson T., A theoretical analysis of the frequency of gaps in plant stands, Agric. Meteorol. 8 (1971) $25-38$.

[36] Pearcy R.W., Photosynthetic utilisation of lightflecks by understory plants, Aust. J. Plant Physiol. 15 (1988) 223-238.

[37] Perttunen J., Sievänen R., Nikinmaa E., LIGNUM: a model combining the strcture and the functioning of trees, Ecol. Model. 108 (1998) 189-198.

[38] Poulson M., Vogelmann T., Epidermal focussing and effects upon photosynthetic light-harvesting in leaves of Oxalis, Plant, Cell and Environ. 13 (1990) 803-811.

[39] Prusinkiewicz P., Modelling of spatial structure and development of plants: a review, Sci. Hortic. 74 (1998) 113-149.

[40] Rapidel B., Étude expérimentale et simulation des transferts hydriques dans les plantes individuelles : application au caféier (Coffea arabica L.), Ph.D. thesis, université de Montpellier II, 1995.

[41] de Reffye P., Édelin C., Franç on J., Jaeger M., Puech C., Plant models faithful to botanical structure 
and development, Comput. Graphics (SIGGRAPH'88 Proceedings) 22 (1988) 151-158.

[42] de Reffye P., Houillier F., Modelling plant growth and architecture: Some recent advances and applications to agronomy and forestry, Curr. Sci. 73 (1997) 984-992.

[43] Ross J. K., Marshak A. L., Calculation of canopy bidirectional reflectance using the Monte Carlo method, Remote Sens. Environ. 24 (1988) 213-225.

[44] Ross J. K., Marshak A. L., The influence of leaf orientation and the specular component of leaf reflectance on the canopy bidirectional reflectance, Remote Sens. Environ. 27 (1989) 251-260.

[45] Sanz C., Espana M., Baret F., Vaillant L., Hanocq J.F., Sarrouy C., Clastre P., Bruguier N., Chelle M., Andrieu B., Zurfluh O., Bidirectional characteristics of leaf reflectance and transmittance: measurement and influence on canopy bidirectional reflectance, in: Guyot G., Phulpin T. (Eds.), Physical Measurements and Signatures in Remote Sensing, Balkema 2, 1997, pp. 583-590.

[46] Schröder P., Hanrahan P., A closed form expression for the form factor between two polygons, Technical report CS-TR-404-93, Department of Computer Science, Princeton University, USA, 1993.
[47] Schröder P., Sweldens W., Spherical wavelets: efficiently representing functions on the sphere, in: Cook R. (Ed.), SIGGRAPH 95 Conference Proceedings, Annual Conference Series, ACM SIGGRAPH, Addison Wesley, Reading, MA, USA, 1995, pp. 161-172.

[48] Sillion F., Arvo J. R., Westin S. H., Greenberg D. P., A global illumination solution for general reflectance distributions, Comput. Graphics (SIGGRAPH '91 Proceedings)25 (1991) 187-196.

[49] Sinoquet H., Rivet P., Drouet J. L., Méthodes de digitalisation et de visualisation 3D de l'architecture des plantes, in: Andrieu B. (Ed.), Séminaire “ Modélisation architecturale ", Paris, 10-12 mars, Inra Département de Bioclimatologie, 1997, pp. 31-37.

[50] Sparrow E. M., On the calculation of radiant interchange between surfaces, in: Ibele W. (Ed.), Modern Developments in Heat Transfer, Academic Press, New York, USA, 1963.

[51] Verhoef W., Earth observation modelling based on layer scattering matrices, Remote Sens. Environ. 17 (1985) 164-178.

[52] Vogelmann T., Martin G., The functional significance of palisade tissue: penetration of directional versus diffuse light, Plant, Cell Environ. 16 (1993) 65-72. 